\title{
Wegner-type Bounds for a Multi-particle Continuous Anderson Model with an Alloy-type External Potential
}

\author{
A. Boutet de Monvel • V. Chulaevsky • P. Stollmann • \\ Y. Suhov
}

Received: 11 October 2008 / Accepted: 18 November 2009 / Published online: 3 December 2009

(C) Springer Science+Business Media, LLC 2009

\begin{abstract}
We consider an $N$-particle quantum systems in $\mathbb{R}^{d}$, with interaction and in presence of a random external alloy-type potential (a continuous $N$-particle Anderson model). We establish Wegner-type bounds (inequalities) for such models, giving upper bounds for the probability that random spectra of Hamiltonians in finite volumes intersect a given set.
\end{abstract}

Keywords Anderson localisation · Wegner-type bounds · Multi-particle systems

\section{Introduction. The $N$-particle Hamiltonian in the Continuum}

This paper is concerned with a multi-particle Anderson model in $\mathbb{R}^{d}$ with interaction. The Hamiltonian $\mathbf{H}\left(=\mathbf{H}^{(N)}(\omega)\right)$ is a Schrödinger operator of the form

$$
\mathbf{H}=-\frac{1}{2} \boldsymbol{\Delta}+\mathbf{U}(\mathbf{x})+\mathbf{V}(\mathbf{x} ; \omega)
$$

acting on functions $\phi \in L_{2}\left(\mathbb{R}^{d \times N}\right)$ where $\mathbb{R}^{d \times N}=\mathbb{R}^{d} \times \cdots \times \mathbb{R}^{d}$ ( $N$ times). This means that we consider $N$ particles, each living in $\mathbb{R}^{d}$, in the following fashion: $\mathbf{x}=\left(x_{1}, \ldots, x_{N}\right) \in \mathbb{R}^{d \times N}$

\footnotetext{
A. Boutet de Monvel

Institut de Mathématiques de Jussieu, Université Paris 7, 175 rue du Chevaleret, 75013 Paris, France e-mail: aboutet@math.jussieu.fr

V. Chulaevsky $(\bowtie)$

Département de Mathématiques, Université de Reims, Moulin de la Housse, B.P. 1039,

51687 Reims Cedex 2, France

e-mail: victor.tchoulaevski@univ-reims.fr

P. Stollmann

Fakultät für Mathematik, Technische Universität Chemnitz, 09107 Chemnitz, Germany

e-mail: peter.stollmann@mathematik.tu-chemnitz.de

Y. Suhov

Statistical Laboratory, DPMMS, University of Cambridge, Wilberforce Road, Cambridge, CB3 0WB, UK

e-mail: yms@statslab.cam.ac.uk
} 
where $x_{j}=\left(\mathrm{x}_{j}^{(1)}, \ldots, \mathrm{x}_{j}^{(d)}\right) \in \mathbb{R}^{d}$ represents the coordinates of the $j$ 's particle. Next, $-\frac{1}{2} \boldsymbol{\Delta}$ is the standard kinetic energy resulting from adding up the kinetic energies $-\frac{1}{2} \Delta_{j}$ of the different particles and assuming that we are dealing with particles of unit mass.

The potential energy term $\mathbf{U}(\cdot)$ is, as usually, identified with the operator of multiplication, by a function denoted by the same symbol. It represents the interaction between particles. More precisely, a fairly standard form is where $\mathbf{U}$ is a sum of one- and two-body terms:

$$
\mathbf{U}(\mathbf{x})=\sum_{1 \leq j \leq N} U^{(1)}\left(x_{j}\right)+\sum_{1 \leq j<j^{\prime} \leq N} U^{(2)}\left(x_{j}, x_{j^{\prime}}\right), \quad \mathbf{x}=\left(x_{1}, \ldots, x_{N}\right),
$$

with function $U^{(1)}$ representing the external potential and function $U^{(2)}$ the potential of interaction between a pair of particles. In fact, in this paper we do not need the above form of $\mathbf{U}$; we only assume

(C0) Boundedness of function $\mathbf{U}$ :

$$
\mathbf{U} \in L^{\infty}\left(\mathbb{R}^{d \times N}\right)
$$

We assume the potential $\mathbf{U}$ to be invariant under translations in the 1-particle space $\mathbb{R}^{d}$ : $\mathbf{x}=\left(x_{1}, \ldots, x_{N}\right) \mapsto\left(x_{1}+y, \ldots, x_{N}+y\right), y \in \mathbb{R}^{d}$. Such an assumption is natural from the physical point of view, and it is required to ensure ergodicity of the ensemble of random Hamiltonians $\mathbf{H}^{(N)}(\omega)$ under group $\mathbb{R}^{d}$. However, we do not use ergodicity in the present paper.

In addition, the particles are subject to a common external random potential $V(x ; \omega)$ where $\omega$ runs through a probability space $\Omega$. The respective potential energy appearing in the Hamiltonian $\mathbf{H}$ results in adding the potential energies of the single particle and thus reads

$$
\mathbf{V}(\mathbf{x} ; \omega)=\sum_{1 \leq j \leq N} V\left(x_{j} ; \omega\right), \quad \mathbf{x}=\left(x_{1}, \ldots, x_{N}\right)
$$

In this paper we consider an alloy-type random potential $V(x ; \omega), x \in \mathbb{R}^{d}$, with specific properties listed in the next section.

For a single-particle system (with $N=1$ ), $\boldsymbol{\Delta}$ is reduced to $\Delta, \mathbf{U}(\mathbf{x})$ to $U^{(1)}(x)$ and $\mathbf{V}(\mathbf{x} ; \omega)$ to $V(x ; \omega)$, with operator $\mathbf{H}$ acting in $L_{2}\left(\mathbb{R}^{d}\right)$. A discrete version of the model arises when $\mathbb{R}^{d}$ is replaced by $\mathbb{Z}^{d}$ (with the Laplacian replaced by its lattice counterpart); it is often called a tight-binding Anderson model. In the tight binding model, both for singleparticle (see $[6,13,20,23]$ and the references therein) and multi-particle (see [3, 8-10]) cases, a large disorder introduced through the random external potential field can generate a pure point spectrum of the Hamiltonian, i.e., Anderson localisation. For a continuous-space Hamiltonian $\mathbf{H}$ as in (1.1), such a phenomenon is expected to occur near the lower edge of the spectrum of $\mathbf{H}$; for recent results in the single-particle case, see, e.g., recent papers [5] and [1].

An important part of known proofs of Anderson localisation is based on estimates of 'regularity' of the eigenvalue distributions for the model in a finite volume. Specifically, the proof based on the multi-scale analysis (MSA) uses the so-called Wegner-type bounds. The bounds are also instrumental in other important problems, viz., in relation to the integrated density of states (IDS). In the present paper we take the first step in showing Anderson localisation in the continuum multi-particle model (near the lower edge of the spectrum of Hamiltonian $\mathbf{H}$ ) by proving suitable Wegner-type bounds for such models; see Theorems 1 
and 2 below. The actual proof of Anderson localisation for these models, is deferred to a future work [4].

The Wegner-type bounds assess concentration of the eigenvalues of $\mathbf{H}_{\Lambda}\left(=\mathbf{H}_{\Lambda}(\omega)\right)$, a finite-volume version of Hamiltonian $\mathbf{H}$ in (1.1). The operator $\mathbf{H}_{\boldsymbol{\Lambda}}$ acts on functions in $L_{2}(\Lambda)$ :

$$
\mathbf{H}_{\boldsymbol{\Lambda}}=-\frac{1}{2} \boldsymbol{\Delta}+\mathbf{U}(\mathbf{x})+\mathbf{V}(\mathbf{x} ; \omega) \quad \text { with Dirichlet boundary conditions on } \partial \mathbf{\Lambda} .
$$

Here $\Lambda \subset \mathbb{R}^{d \times N}$ is a $(d \times N)$-dimensional cube which we call an (N-particle) box, of the form $\boldsymbol{\Lambda}=\Lambda^{(1)} \times \cdots \times \Lambda^{(N)}$ ( $N$ times) where $\Lambda \subset \mathbb{R}^{d}$ is a cube with edges parallel to the coordinate axes in $\mathbb{R}^{d}$. To be more specific, given $L>0$ and $v=\left(\mathrm{v}^{(1)}, \ldots, \mathrm{v}^{(d)}\right) \in \mathbb{R}^{d}$, we set henceforth:

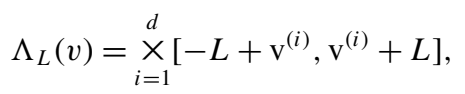

and, for $\mathbf{u}=\left(u_{1}, \ldots, u_{N}\right) \in \mathbb{R}^{d \times N}$,

$$
\boldsymbol{\Lambda}_{L}(\mathbf{u})=\Lambda_{L}\left(u_{1}\right) \times \cdots \times \Lambda_{L}\left(u_{N}\right) .
$$

For sets $\boldsymbol{\Lambda}_{L}(\mathbf{u})$ of the above form we define coordinate projections $\Pi_{j} \boldsymbol{\Lambda}_{L}(\mathbf{u}), 1 \leq j \leq n$, by

$$
\Pi_{j} \Lambda_{L}(\mathbf{u})=\Lambda_{L}\left(u_{j}\right) \subset \mathbb{R}^{d} .
$$

A slightly more general case of a parallelepiped $\Lambda_{L_{1}}\left(u_{1}\right) \times \cdots \times \Lambda_{L_{N}}\left(u_{N}\right)$, with arbitrary lengths $L_{j}$, can be treated in a similar way.

We would like to note that the methods adopted in this paper are applicable to a wide range of other boundary conditions including periodic and 'elastic' (e.g., Neumann's). In fact, what we need is that operator $\mathbf{H}_{\Lambda}$ in (1.4) is self-adjoint and has a compact resolvent $\left(\mathbf{H}_{\boldsymbol{\Lambda}}-\mathrm{z} I\right)^{-1}$ for non-real $\mathrm{z} \in \mathbb{C} \backslash \mathbb{R}$; this covers all 'classical' Krein's self-adjoint extensions, from the 'soft' (Neumann's) to 'hard' (Dirichlet's).

Throughout the text, the short-hand notation $\boldsymbol{\Lambda}$ is used for a box $\boldsymbol{\Lambda}_{L}(\mathbf{u})$, when the parameters $L$ and $\mathbf{u}$ are unambiguous. Similarly, $\boldsymbol{\Lambda}^{\prime}$ stands for $\boldsymbol{\Lambda}_{L^{\prime}}\left(\mathbf{u}^{\prime}\right)$. We will also use an alternative notation $\Pi_{j} \boldsymbol{\Lambda}$ instead of $\Lambda_{L}\left(u_{j}\right)$ and $\Pi_{j} \boldsymbol{\Lambda}^{\prime}$ instead of $\Lambda_{L_{j}^{\prime}}\left(u_{j}^{\prime}\right)$, meaning that $\Pi_{j}$ stands for the projection onto the $j$ th factor $\mathbb{R}^{d}$ in the Cartesian product $\mathbb{R}^{d \times N}$, where $j=1, \ldots, N$.

Under the conditions imposed in this paper, the operator $\mathbf{H}_{\Lambda}$ has a compact resolvent and therefore has purely discrete spectrum consisting of eigenvalues of finite multiplicity. It is convenient to write these eigenvalues $E^{(\boldsymbol{\Lambda})}=E^{(\boldsymbol{\Lambda})}(\omega)$ in increasing order:

$$
E_{0}^{(\boldsymbol{\Lambda})} \leq E_{1}^{(\boldsymbol{\Lambda})} \leq E_{2}^{(\boldsymbol{\Lambda})} \leq \cdots
$$

The 'one-volume' Wegner-type bound is an upper bound for the probability

$$
\mathbb{P}\left(\exists k \text { with }\left|E-E_{k}^{(\boldsymbol{\Lambda})}\right| \leq \epsilon\right),
$$

that at least one eigenvalue $E_{k}^{(\boldsymbol{\Lambda})}$ of the operator $H_{\boldsymbol{\Lambda}}$ falls in a (narrow) interval around a given point $E$ on the spectral axis. See Theorem 1 in Sect. 3 below. The 'two-volume' Wegner-type bound is an upper bound for the probability

$$
\mathbb{P}\left(\exists k \text { and } k^{\prime} \text { with } E_{k}^{(\boldsymbol{\Lambda})}, E_{k^{\prime}}^{\left(\boldsymbol{\Lambda}^{\prime}\right)} \in I \text { and }\left|E_{k}^{(\boldsymbol{\Lambda})}-E_{k^{\prime}}^{\left(\boldsymbol{\Lambda}^{\prime}\right)}\right| \leq \epsilon\right) .
$$


This is the probability that some eigenvalues $E_{k}^{(\boldsymbol{\Lambda})}$ and $E_{k^{\prime}}^{\left(\boldsymbol{\Lambda}^{\prime}\right)}$ of the operators $\mathbf{H}_{\boldsymbol{\Lambda}}$ and $\mathbf{H}_{\boldsymbol{\Lambda}^{\prime}}$ come close to each other in a given interval $I \subset \mathbb{R}$ of the spectral axis, for two boxes, $\boldsymbol{\Lambda}$ and $\boldsymbol{\Lambda}^{\prime}$, which satisfy a condition of 'separability'; see Definition 2 and Theorem 2 in Sect. 4. Here and below, $\mathbb{P}$ stands for the corresponding probability measure on the underlying probability space $\Omega$ (see below).

Remarks (1) From the probabilistic point of view, bounds for probabilities (1.8) and (1.9) are examples of concentration inequalities, albeit for rather implicit random variables $E_{k}^{(\boldsymbol{\Lambda})}$ and $E_{k^{\prime}}^{\left(\Lambda^{\prime}\right)}$ carrying a considerable amount of dependence. The nature of these bounds is that they decrease to 0 when $\epsilon \rightarrow 0$ but increase with $\Lambda$ and $\boldsymbol{\Lambda}^{\prime}$; a convenient form is where the bounds are products comprising 'volume factors' and a 'width factor'; cf. (3.1) and (4.7).

For single-particle Anderson models, under natural assumptions on the character of the random terms in Hamiltonians (1.1) and (1.4), the Wegner bounds (both one-volume and two-volume) are rather straightforward. We do not provide here a full (and rather extensive) bibliography on single-particle Wegner's bounds; apart from paying tribute to the original work by Wegner [25]. Instead, we refer the reader to the monographs [6, 20, 23], the surveys $[18,24]$ and the recent papers $[2,11]$ where such bibliography is presented.

(2) For an $N$-particle tight binding model (beginning with $N=2$ ), a version of onevolume and two-volume Wegner bounds suitable for the MSA was established in [8]; different versions of one-volume bounds (but not two-volume bounds) can also be found in [17].

(3) The fairly simple method of proof used in this paper does not yield the optimal volume factors in (3.1) and (4.7). In the preprint [26] (incorporated into the later paper [KlZ]), a more subtle (and technically more involved) method is used giving an optimal volume factor in the one-volume bound (3.1), albeit under different conditions. Cf. Remark (3) after the statement of our Theorem 1 in Sect. 3. However, in our opinion, optimality of the volume factor in (3.1) (and (4.7)) is an issue of minor importance at the moment. In fact, in [19] one can find an abstract reason (not involving finite volume estimates) for continuity of the IDS. On the other hand, it should be emphasised that the existing methods of proving Anderson localisation for $N$ particles require both one-volume and two-volume multi-particle Wegnertype bounds.

(4) The aforementioned condition of separability of boxes $\boldsymbol{\Lambda}$ and $\boldsymbol{\Lambda}^{\prime}$ used for assessing the probability in (1.9) is introduced in Definition 2, Sect. 4. It follows Definition 1.2 from [10] (where a tight binding model was considered) and serves a similar purpose: to provide 'enough randomness' for a concentration bound argument.

(5) The methods of proof employed in this paper are similar to those from $[8,10]$ used for tight binding models. However, the specifics of continuous models required some additional arguments making it impossible to simply refer to papers $[8,10]$.

(6) Interaction potentials $\mathbf{U}(\mathbf{x})$ with a 'hard core' component can also be considered; this requires only a minor modification of our arguments.

In the next section, we give formal conditions upon the structure of the potential energy term in (1.3). Throughout the paper, $|\boldsymbol{\Lambda}|$ denotes the (Euclidean) volume of box $\boldsymbol{\Lambda}$ in $\mathbb{R}^{d \times N}$. Similarly, $\left|\Pi_{j} \boldsymbol{\Lambda}\right|$ stands for the volume of the projection $\Pi_{j} \boldsymbol{\Lambda}$ in $\mathbb{R}^{d}$ and $\left|\bigcup_{j \in \mathcal{J}} \Pi_{j} \boldsymbol{\Lambda}\right|$ for the volume of the union $\bigcup_{j \in \mathcal{J}} \Pi_{j} \boldsymbol{\Lambda}$ where $\mathcal{J}$ is a non-empty subset of $\{1, \ldots, N\}$. We also use the notation \# $J$ for the cardinality of a (finite) set $J$ encountered in the argument below, in particular, a finite collection of lattice sites of the form $\Pi_{j} \Lambda \cap \mathbb{Z}^{d}$ or their unions. Here

$$
\mathbb{Z}^{d}=\left\{s=\left(\mathrm{s}^{(1)}, \ldots, \mathrm{s}^{(d)}\right): \mathrm{s}^{(i)} \in \mathbb{Z}, i=1, \ldots, d\right\}
$$


is the integer lattice canonically embedded in $\mathbb{R}^{d}$. We will always assume that cubes $\boldsymbol{\Lambda}=$ $\boldsymbol{\Lambda}_{L}(\mathbf{u})$ have the sidelength $2 L \geq 1$, so that $\boldsymbol{\Lambda}$ contains at least one integer point. Moreover, when $2 L \geq 1$, the ratio between the volume $\left|\Pi_{j} \boldsymbol{\Lambda}\right|$ and the cardinality $\#\left(\Pi_{j} \boldsymbol{\Lambda} \cap \mathbb{Z}^{d}\right)$ is bounded from above and below by positive constants.

\section{External Alloy-type Potentials}

In this work, the random external potential $V(x ; \omega), x \in \mathbb{R}^{d}, \omega \in \Omega$, is assumed to be of an alloy-type, over a cubic lattice:

$$
V(x ; \omega)=\sum_{s \in \mathbb{Z}^{d}} \mathrm{~V}_{s}(\omega) \varphi(x-s) .
$$

Here $\mathbf{V}=\left(\mathrm{V}_{s}, s \in \mathbb{Z}^{d}\right)$, is a family of real random variables $\mathrm{V}_{s}$ on some probability space $(\Omega, \mathfrak{B}, \mathbb{P})$. In probabilistic terms, $\mathbf{V}$ is a real-valued random field on $\mathbb{Z}^{d}$. Further, $\varphi$ is a (nonrandom) 'bump function' $y \in \mathbb{R}^{d} \mapsto \varphi(y)$.

Physically speaking, the random variables $\mathrm{V}_{s}$ represents the amplitude of an 'impurity' at site $s$ of the lattice $\mathbb{Z}^{d}$ while the function $\varphi$ describes the 'propagation' of the impact of this impurity across the space $\mathbb{R}^{d}$. The form of the potential $V(x ; \omega)$ specified in $(2.1)$ reflects the presence of 'heavy' atoms (ions) attached to sites of a crystal lattice.

To avoid excessive technicalities concerning operators $\mathbf{H}_{\boldsymbol{\Lambda}}$ we assume that the bump function satisfies

(C1) Boundedness condition: $\varphi$ is a bounded non-negative function, with

$$
\sup _{x \in \mathbb{R}^{d}}\left[\sum_{s \in \mathbb{Z}^{d}} \varphi(x-s)\right]<+\infty, \quad \forall x \in \mathbb{R}^{d} .
$$

We will also need a lower bound:

(C2) Covering condition:

$$
\sum_{s \in \Lambda_{L}(u) \cap \mathbb{Z}^{d}} \varphi(x-s) \geq 1, \quad \forall L \geq 1, u \in \mathbb{R}^{d}, x \in \Lambda_{L}(u) .
$$

Finally, it is helpful to assume

(C3) Finite propagation range condition: the function $\varphi$ has bounded support: $\exists R \in$ $(0, \infty)$ with

$$
\varphi(y)=0 \quad \text { whenever } y=\left(\mathrm{y}^{(1)}, \ldots, \mathrm{y}^{(d)}\right) \text { has } \max _{1 \leq i \leq d}\left|\mathrm{y}^{(j)}\right|>R .
$$

Remark A further generalisation occurs if we assume that the bump function changes its shape for various sites of $\mathbb{Z}^{d}$, i.e., we replace $\varphi(x-s)$ with $\varphi_{s}(x-s)$ in (2.1): Conditions (C1) and (C2) are then re-written in an obvious fashion. It is also possible to incorporate random displacements for sites $s \in \mathbb{Z}^{d}$, taking, instead of $\varphi(x-s)$, a summand $\varphi_{s}(x-s+$ $Z_{s}$ ) where $Z_{s}=\left(Z_{s}^{(1)}, \ldots, Z_{s}^{(d)}\right)$ is a random variable with values in $\mathbb{R}^{d}$ (for definiteness, one may assume that $\left.\max _{1 \leq i \leq d}\left|Z_{s}^{(i)}\right|<1 / 2\right)$; the random field $\mathbf{Z}=\left(Z_{s}, s \in \mathbb{Z}^{d}\right)$ may have a fairly general joint distribution with $\mathbf{V}$ satisfying some mild conditions. 
Now we discuss the properties of the random field $\mathbf{V}$ needed in this paper. To begin with, it is convenient to assume

(C4) Boundedness condition:

$$
\sup _{s \in \mathbb{Z}^{d}} \sup _{\mathbf{V}} \operatorname{ess}\left|\mathrm{V}_{s}\right|=: M<\infty .
$$

Here and below, sup ess indicates the essential supremum relative to the distribution of random field $\mathbf{V}$ or its sub-field $\mathbf{V}_{\{s\}^{\mathrm{c}}}=\left(V_{t}, t \in \mathbb{Z}^{d} \backslash\{s\}\right)$, according to the specification provided in the equation under consideration.

Next, given a site $s \in \mathbb{Z}^{d}$, consider the conditional distribution function

$$
F_{s}\left(\mathrm{y} \mid \mathfrak{B}_{\{\mathrm{s}\}^{\mathrm{c}}}\right):=\mathbb{P}\left(\mathrm{V}_{\mathrm{s}}<\mathrm{y} \mid \mathfrak{B}_{\{\mathrm{s}\}}\right)
$$

relative to the sigma-algebra $\mathfrak{B}_{\{s\}^{c}}$ generated by $\mathbf{V}_{\{s\}^{c}}$. Then, given $\epsilon>0$, we set:

$$
v(\epsilon):=\sup _{s \in \mathbb{Z}^{d}} \sup _{\mathrm{y} \in \mathbb{R}} \sup _{\mathbf{V}_{\{s\}^{\mathrm{c}}}} \operatorname{ess}\left[F_{s}\left(\mathrm{y}+\epsilon \mid \mathfrak{B}_{\{s\}^{\mathrm{c}}}\right)-F_{s}\left(\mathrm{y} \mid \mathfrak{B}_{\{s\}^{\mathrm{c}}}\right)\right] .
$$

The Wegner-type bounds derived in this paper (see (3.1) and (4.7) below) contain the factor $\nu(\epsilon)$; formally speaking, these bounds remain valid without any further assumptions on $v(\epsilon)$. (Of course, the inequality $v(\epsilon) \leq 1$ holds trivially as $F_{s}\left(\cdot \mid \mathfrak{B}_{\{s\}}\right.$ ) is a distribution function.) However, the use of these inequalities for establishing Anderson localisation is based on the assumption that $v(\epsilon) \rightarrow 0$ as $\epsilon \rightarrow 0$, and this convergence is fast enough. Namely, for a proof of Anderson localisation in the framework of the MSA, it is usually required to assume that $\nu(\epsilon) \leq C \epsilon^{b}$, for some $b, C \in(0, \infty)$ (Hölder-continuity of the conditional marginal distributions), or, at least, $v(\epsilon) \leq C \ln ^{-A} \epsilon^{-1}$, for some $C<\infty$, and sufficiently large $A>0$ (log-Hölder-continuity).

Remarks (1) This approach allows us to cover a large class of random fields $\mathbf{V}$ (such as a 'nice' bounded function of a regular Gaussian random field as well as some Gibbsian random fields). We refer the reader to [7] for a more detailed discussion of correlated potentials.

(2) We stress the fact that in Condition (C4) we do not need independence of the variables $\mathrm{V}_{s}$ for different sites $s \in \mathbb{Z}^{d}$. However, the quantity $\nu(\epsilon)$ can easily be understood in the particular case of independent random variables $\mathrm{V}_{s}$. Denote by $\mu_{s}=\mathbb{P} \circ \mathrm{V}_{\mathrm{s}}$ the law of $\mathrm{V}_{s}$ which is a measure on the real line and by $c\left(\mu_{s} ; \epsilon\right)$ its modulus of continuity, i.e.,

$$
c\left(\mu_{s} ; \epsilon\right)=\sup _{a \in \mathbb{R}} \mu_{s}([a, a+\epsilon]) .
$$

In this case,

$$
v(\epsilon)=\sup _{s \in \mathbb{Z}^{d}} c\left(\mu_{s} ; \epsilon\right) .
$$

(3) Condition (C0) (see (1.2)) as well as (C1)-(C2) are stronger than what is needed in our argument: in reality we only need to have some control on the number of eigenvalues of $\mathbf{H}_{\Lambda}$ below a given energy threshold.

(4) The regularity condition imposed in [2] and called there Assumption R also yields $\nu(\epsilon) \leq \rho \epsilon$ where $\rho \in(0, \infty)$ is a constant.

(5) For measure-theoretic concepts used above, see, e.g., [12], Appendix; [14], Theorem 3.1; [15], Chap. V; [16], Chap. 4. 


\section{A One-volume Wegner-type Bound}

The one-volume Wegner-type bound for Hamiltonians $\mathbf{H}_{\boldsymbol{\Lambda}}$ is given in Theorem 1 below. Let $\Sigma\left(\mathbf{H}_{\boldsymbol{\Lambda}}\right)$ denote the (random) spectrum of (random) operator $\mathbf{H}_{\boldsymbol{\Lambda}}$, i.e., the countable set

$$
\Sigma\left(\mathbf{H}_{\boldsymbol{\Lambda}}(\omega)\right)=\left\{E_{k}^{(\boldsymbol{\Lambda})}(\omega): k=0,1, \ldots\right\}
$$

of its eigenvalues.

Theorem 1 Assume that Conditions (C0) (Sect. 1) and (C1)-(C4) (Sect. 2) are satisfied; see (1.2), (2.2)-(2.4), (2.5). Let $\Lambda \subset \mathbb{R}^{d \times N}$ be a parallelepiped of the form (1.6). Then there is a constant $C_{1}>0$ such that for any box $\boldsymbol{\Lambda}$, all $E \in \mathbb{R}$ and $\epsilon \in(0,1)$ :

$$
\mathbb{P}\left([E, E+\epsilon] \cap \Sigma\left(H_{\boldsymbol{\Lambda}}\right) \neq \emptyset\right) \leq C_{1}(1+E \vee 0)^{d / 2}\left|\mathbf{\Lambda} \| \Pi_{1} \mathbf{\Lambda}\right| v(\epsilon)
$$

Remarks (1) The expression in the RHS of (3.1) includes the volume factors $|\boldsymbol{\Lambda}|$ and $\left|\Pi_{1} \boldsymbol{\Lambda}\right|$ with different meaning. The first one, $|\boldsymbol{\Lambda}|$, together with $C(1+E \vee 0)^{d / 2}$, comes from an upper bound of the number of eigenvalues of $\mathbf{H}_{\Lambda}$ to the left of point $E+1$. The second one, $\left|\Pi_{1} \boldsymbol{\Lambda}\right|$ together with $v(\epsilon)$, comes from the concentration bound for each individual eigenvalue based on [7, 22, 23] which we summarise in Lemma 1 below.

(2) In a more general situation where $\boldsymbol{\Lambda}$ is a parallelepiped $\Lambda_{L_{1}}\left(u_{1}\right) \times \cdots \times \Lambda_{L_{N}}\left(u_{N}\right)$ with arbitrary sidelengths $L_{1}, \ldots, L_{N}>0$, the factor $\left|\Pi_{1} \boldsymbol{\Lambda}\right|$ in (3.1) can be replaced by $\min _{1 \leq j \leq N}\left|\Pi_{j} \boldsymbol{\Lambda}\right|$.

(3) The one-volume Wegner bound obtained in [26, p. 7], has an expression $C_{1}|\boldsymbol{\Lambda}| \epsilon$ in the right-hand side (RHS) which is better than the RHS of our (3.1). (Traditionally, bounds similar to (3.1), with the RHS $C|\mathbf{\Lambda}| \epsilon$, are called Wegner's bounds; to emphasise the difference we use in this paper a more general term 'Wegner-type bounds'.) In addition, the class of interaction potentials considered in [26] is notably larger than that in this paper (for instance, [26] deals with a Coulomb potential). However, the assumptions introduced in [26] about the external potential random field include a requirement that the random variables $\mathrm{V}_{s}$ are independent and identically distributed and have a bounded common probability-density function (PDF). [The author of [26] speaks of a 'price' to be paid for the Wegner bound with the RHS $C|\boldsymbol{\Lambda}| \epsilon$.] Furthermore, as we noted earlier, the proofs in [26] are technically more involved and use specific properties of the Laplacian. On the contrary, the method used in this paper requires only a bound for the number of the eigenvalues of the kinetic energy operator in a given interval.

As was said before, for our purpose (Anderson localisation in the continuum multiparticle alloy-type model near the lower edge of the spectrum), the estimate (3.1), together with its two-volume counterpart, (4.7), suffices, provided that $v(\epsilon) \rightarrow 0$ as $\epsilon \rightarrow 0$ fast enough. More precisely, it is not crucial whether the RHS in (3.1) contains just a volumelike factor $|\boldsymbol{\Lambda}|$ or the product $|\boldsymbol{\Lambda}|\left(\min _{1 \leq j \leq N}\left|\Pi_{j} \boldsymbol{\Lambda}\right|\right)$ : such changes results in straightforward modifications the MSA inductive scheme. The same is true about the (seemingly more intricate) RHS of the two-volume Wegner-type bound in (4.7).) Replacing $\epsilon$ with the continuity modulus $v(\epsilon)$ is our 'price' for considering distributions without a PDF. As was said before, [26] does not contain two-volume Wegner-type bounds (and it remains to be seen whether a suitable two-volume Wegner-type bound holds under the conditions adopted in [26]).

(3) We do not address in this paper the issue of optimality of bounds in (3.1) and (4.7).

Following [7], we introduce 
Definition 1 Consider $\mathbb{R}^{q}$ and its positive orthant $\mathbb{R}_{+}^{q}$. A function $\Phi: \mathbb{R}^{q} \rightarrow \mathbb{R}$ is called diagonally-monotone (DM) if

(i) $\Phi(\mathbf{w}+\mathbf{r}) \geq \Phi(\mathbf{w}) \forall \mathbf{r} \in \mathbb{R}_{+}^{q}$ and any $\mathbf{w} \in \mathbb{R}^{q}$,

(ii) $\Phi(\mathbf{w}+t \mathbf{e})-\Phi(\mathbf{w}) \geq t, \forall \mathbf{w} \in \mathbb{R}^{q}$ and $t>0$, where $\mathbf{e}=(1,1, \ldots, 1) \in \mathbb{R}^{q}$.

Lemma 1 Let $J$ be a finite set with $q=\# J \geq 2$, and $\mu$ be a probability measure on $\mathbb{R}^{J}$. For every $j \in J$, denote by $\mu^{(j)}\left(\cdot \mid w_{\neq j}\right)$ the conditional probability measure induced by $\mu$ on the $j$-th coordinate $\mathrm{w}^{(j)}$ conditioned on $w_{\neq j}$. Set:

$$
\sigma(\mu, \epsilon)=\sup _{\mathbf{w}_{\neq j}} \operatorname{ess} \max _{j=1, \ldots, q} \sup _{a \in \mathbb{R}}\left[\mu^{(j)}\left([a, a+\epsilon] \mid \mathbf{w}_{\neq j}\right)\right] .
$$

Let the function $\Phi: \mathbb{R}^{J} \rightarrow \mathbb{R}$ be DM. Then for all $\epsilon>0$, any open interval $I \subset \mathbb{R}$ of length $\epsilon$,

$$
\mu\{\mathbf{w}: \Phi(\mathbf{w}) \in I\} \leq q \cdot \sigma(\mu, \epsilon)
$$

Proof (Cf. [8, 22, 23]). Here we follow closely the proof given in [7] for a wide class of measures $\mu$. Denote by $\mu_{j}$ the marginal probability induced by $\mu$ on $\mathbb{R}^{J \backslash j j\}}$. The main ingredient is the fact that with the notation above

$$
\mu(A)=\int \mathrm{d} \mu_{j}\left(\widetilde{\mathbf{w}}_{\neq j}\right) \mathrm{d} \mu^{(j)}\left(\mathrm{w}^{(j)} \mid \widetilde{\mathbf{w}}_{\neq j}\right) \mathbf{1}\left(\left(\widetilde{\mathrm{w}}^{(1)}, \ldots, \mathrm{w}^{(j)}, \ldots, \widetilde{\mathrm{w}}^{(q)}\right) \in A\right)
$$

for any measurable set $A \subseteq \mathbb{R}^{J}$ and any $j \in J$ by the very definition of conditional probabilities and marginals.

Take $I=(a, b)$ where $b-a=\epsilon$ and consider the set

$$
A=\{\mathbf{w}: \Phi(\mathbf{w}) \leq a\} .
$$

Define sets $A_{j}^{\epsilon}, j=0, \ldots, q$ :

$$
A_{0}^{\epsilon}=A, \quad A_{j}^{\epsilon}=A_{j-1}^{\epsilon}+[0, \epsilon] \mathbf{e}_{j}:=\left\{\mathbf{w}+t \mathbf{e}_{j}: \mathbf{w} \in A_{j-1}^{\epsilon}, t \in[0, \epsilon]\right\},
$$

with $A_{1}^{\epsilon} \subset \cdots \subset A_{q}^{\epsilon}$. By virtue of the DM property: $\{\mathbf{w}: \Phi(\mathbf{w})<b\} \subset A_{q}^{\epsilon}$. Indeed, if $\Phi(\mathbf{w})<b$, then for the vector $\mathbf{w}^{\prime}:=\mathbf{w}-\epsilon \cdot \mathbf{e}$ we have:

$$
\Phi\left(\mathbf{w}^{\prime}\right) \leq \Phi\left(\mathbf{w}^{\prime}+\epsilon \cdot \mathbf{e}\right)-\epsilon=\Phi(\mathbf{w})-\epsilon \leq b-\epsilon \leq a,
$$

meaning that $\Phi\left(\mathbf{w}^{\prime}\right) \leq a$, i.e., $\mathbf{w}^{\prime} \in A$. Therefore, $\mathbf{w}=\mathbf{w}^{\prime}+\epsilon \cdot \mathbf{e} \in A_{q}^{\epsilon}$. We conclude that

$$
\{\mathbf{w}: \Phi(\mathbf{w}) \in I\}=\{\mathbf{w}: \Phi(\mathbf{w})<b\} \backslash\{\mathbf{w}: \Phi(\mathbf{w}) \leq a\} \subset A_{m}^{\epsilon} \backslash A .
$$

Now write:

$$
\mu\{\mathbf{w}: \Phi(\mathbf{w}) \in I\} \leq \mu\left(A_{q}^{\epsilon} \backslash A\right)=\mu\left(\bigcup_{j=1}^{q}\left(A_{j}^{\epsilon} \backslash A_{j-1}^{\epsilon}\right)\right) \leq \sum_{j=1}^{q} \mu\left(A_{j}^{\epsilon} \backslash A_{j-1}^{\epsilon}\right) .
$$

Given $j=1, \ldots, q$, denote by $\mu^{(\neq j)}$ the marginal distribution of components $\mathrm{v}_{k}$ with $k \neq j$ Next, for $\widetilde{\mathbf{w}}=\left(\widetilde{\mathrm{v}}_{1}, \ldots, \widetilde{\mathrm{v}}_{q-1}\right) \in \mathbb{R}^{q-1}$, introduce combined vectors

$$
(\widetilde{\mathbf{w}}, \mathrm{w})^{(j)}=\left(\widetilde{\mathrm{v}}_{1}, \ldots, \widetilde{\mathrm{v}}_{j-1}, \mathrm{w}, \widetilde{\mathrm{v}}_{j+1}, \ldots, \widetilde{\mathrm{v}}_{q-1}\right) \in \mathbb{R}^{q}, \quad j=1, \ldots, q-1,
$$


with obvious modifications for $j=1$ and $j=q$. Next, set:

$$
I_{j}(\widetilde{\mathbf{w}})=\left\{\mathrm{w} \in \mathbb{R}:(\mathbf{w}, \mathrm{w})^{(\mathrm{j})} \in \mathrm{A}_{1}^{\epsilon} \backslash \mathrm{A}\right\} .
$$

By definition of $A_{j}^{\epsilon}$, set $I_{j}(\widetilde{\mathbf{w}})$ is an interval of length $\leq \epsilon$. Thus,

$$
\mu\left(A_{1}^{\epsilon} \backslash A\right)=\int \mathrm{d} \mu_{1}(\widetilde{\mathbf{w}}) \int_{I_{1}(\widetilde{\mathbf{w}})} \mathrm{d} \mu^{(1)}(\mathrm{w} \mid \widetilde{\mathbf{w}}) \leq \sigma(\mu, \epsilon) .
$$

Similarly, for $j=2, \ldots, p$ we obtain $\mu\left(A_{j}^{\epsilon} \backslash A_{j-1}^{\epsilon}\right) \leq \sigma(\mu, \epsilon)$. Together with (3.4), this yields (3.3).

Proof of Theorem 1 Our assumptions on the potential term imply that $\mathbf{U}(\cdot)+\mathbf{V}(\cdot ; \omega)$ is uniformly bounded. It is worth mentioning that the upper bound depends upon the range of propagation $R>0$ of the bump functions $\varphi$. Indeed, a given point $x \in \mathbb{R}^{d}$ lies in the support of $O\left(R^{d}\right)$ sites $s \in \mathbb{Z}^{d} \subset \mathbb{R}^{d}$ supporting the bump functions $\varphi(\cdot-s)$, so that the value of the external random potential is a sum of $O\left(R^{d}\right)$ uniformly bounded terms. By the Weyl's law [21], not more than $\widetilde{C}_{1}(1+E \vee 0)^{\frac{d}{2}}|\boldsymbol{\Lambda}|$ eigenvalues of $H_{\boldsymbol{\Lambda}}$ lie below $E+\epsilon$, where $\widetilde{C}_{1}=\widetilde{C}_{1}(R) \in(0, \infty)$ is a constant.

Therefore,

$$
\mathbb{P}\left([E, E+\epsilon] \cap \Sigma\left(H_{\boldsymbol{\Lambda}}\right) \neq \emptyset\right) \leq \sum_{k \leq C_{1}(1+E \vee 0)^{\frac{d}{2}}} \mathbb{P}\left(E_{k}^{(\boldsymbol{\Lambda})} \in[E, E+\epsilon]\right) .
$$

Note that the number of summands in the RHS of (3.5) is proportional to the volume $|\boldsymbol{\Lambda}|$. We will now proceed to prove that every term in the sum in the RHS of (3.5) can be estimated by $\left|\Pi_{1} \boldsymbol{\Lambda}\right| \cdot v(\epsilon)$, leading to the desired bound in (3.1). Fix $k$ and set $J=\Pi_{1} \boldsymbol{\Lambda} \cap \mathbb{Z}^{d}$. We will show that the conditional probability for the $k$ th eigenvalue to fall in $[E, E+\epsilon]$ is bounded:

$$
\sup _{\mathfrak{B}_{\mathbb{Z}^{d} \backslash J} \operatorname{ess}} \mathbb{P}\left(E_{k}^{(\boldsymbol{\Lambda})} \in[E, E+\epsilon] \mid \mathfrak{B}_{\mathbb{Z}^{d} \backslash J}\right) \leq(\# J) \cdot v(\epsilon) .
$$

Here $\mathfrak{B}_{\mathbb{Z}^{d} \backslash J}$ stands for the sigma-subalgebra of $\mathfrak{B}$ generated by $\mathbf{V}_{\mathbb{Z}^{d} \backslash J}=\left\{\mathrm{V}_{s}, s \in \mathbb{Z}^{d} \backslash J\right\}$.

We now aim to use the concentration bound from Lemma 1 above, for a fixed realisation $\mathbf{V}_{\mathbb{Z}^{d} \backslash J}$. Here $\mu$ is identified with $\mathbb{P}_{J}\left(\cdot \mid \mathbf{V}_{\mathbb{Z}^{d} \backslash J}\right)$, the restriction of the conditional distribution $\mathbb{P}\left(\cdot \mid \mathfrak{B}_{\mathbb{Z}^{d} \backslash J}\right)$ to the 'complementary' sigma-algebra $\mathfrak{B}_{J}$ generated by $\mathbf{V}_{J}=\left\{\mathrm{V}_{s}: s \in J\right\}$, conditional on $\mathbf{V}_{\mathbb{Z}^{d} \backslash J}$. Then the quantity $c(\mu ; \epsilon)$ is bounded by $\nu(\epsilon)$ (this follows from the definition of $v(\epsilon)$ in (2.7).

By our definition, $\# J=\#\left(\Pi_{1} \mathbf{\Lambda}\right)$. After setting $\Phi_{k}\left(\mathbf{V}_{J}\right):=E_{k}^{(\boldsymbol{\Lambda})}$, it remains to prove that $\Phi_{k}$ is a DM function. Recall, we are working with eigenvalues of the operator $\mathbf{H}_{\Lambda}$ defined in (1.4) and acting in $L^{2}(\boldsymbol{\Lambda})$, with the external potential term $\mathbf{V}(\mathbf{x} ; \omega)$ of the form (1.3), (2.1). Since the function $\varphi$ is nonnegative (see Condition (C1)), operator $\mathbf{H}_{\boldsymbol{\Lambda}}$ is DM in the variables $V_{s}, s \in J$. Now $\Phi_{k}\left(\mathbf{V}_{J}+t \cdot \mathbf{e}_{J}\right)$ is the $k$-th eigenvalue of

$$
\begin{aligned}
& -\frac{1}{2} \boldsymbol{\Delta}+\mathbf{U}(\mathbf{x})+\left[\sum_{1 \leq j \leq N} \sum_{s \notin J} \mathrm{~V}_{s} \varphi\left(x_{j}-s\right)\right]+\left[\sum_{1 \leq j \leq N} \sum_{s \in J}\left(\mathrm{~V}_{s}+t\right) \varphi\left(x_{j}-s\right)\right] \\
& =\mathbf{H}_{\boldsymbol{\Lambda}}(\omega)+\left[\sum_{1 \leq j \leq N} \sum_{s \in J} t \varphi\left(x_{j}-s\right)\right], \quad \mathbf{x} \in \mathbf{\Lambda} .
\end{aligned}
$$


Here, each term in square brackets represents a multiplication operator, by the corresponding function. From Condition (C2), we know that the corresponding multiplication operator is bounded below by $t \mathbf{I}$, where $\mathbf{I}$ stands for the identity operator. Then the min-max principle for the eigenvalues gives that $\Phi_{k}\left(\mathbf{V}_{J}+t \cdot \mathbf{e}_{J}\right) \geq \Phi_{k}\left(\mathbf{V}_{J}\right)+t$ where $\mathbf{e}_{J}=(1, \ldots, 1) \in \mathbb{R}^{J}$. Therefore, $\Phi_{k}$ is a DM function. Thus, Lemma 1 applies and we get bound (3.1).

Remarks (1) As was mentioned above, our one-volume Wegner-type bound has precursors concerning discrete Schrödinger operators; see [7, 8, 17].

(2) The recent work [2] addresses Wegner-type bounds for correlated potentials in the discrete setting. There the focus is on joint distributions of eigenvalues, not on multi-particle models.

\section{A Two-volume Wegner-type Bound}

In this section we prove the two-volume Wegner-type bound; see Theorem 2 below. As was mentioned earlier, the two-volume Wegner-type bound is established for a pair of separated boxes $\boldsymbol{\Lambda}=\boldsymbol{\Lambda}_{L}(\mathbf{u})$ and $\boldsymbol{\Lambda}^{\prime}=\boldsymbol{\Lambda}_{L^{\prime}}\left(\mathbf{u}^{\prime}\right)$. Recall, this bound assesses the probability that the distance between the spectra $\Sigma\left(H_{\boldsymbol{\Lambda}}\right)$ and $\Sigma\left(H_{\mathbf{\Lambda}^{\prime}}\right)$ of operators $H_{\boldsymbol{\Lambda}}$ and $H_{\boldsymbol{\Lambda}^{\prime}}$ in a given interval $I$ is small; cf. (1.9).

Given $L>0$ and $\mathbf{u}=\left(u_{1}, \ldots, u_{N}\right) \in \mathbb{R}^{d \times N}$ where $u_{j}=\left(\mathrm{u}_{j}^{(1)}, \ldots, \mathrm{u}_{j}^{(d)}\right) \in \mathbb{R}^{d}$, consider the 'extension' of box $\boldsymbol{\Lambda}=\boldsymbol{\Lambda}_{L}(\mathbf{u})$ :

$$
\widehat{\boldsymbol{\Lambda}}=\boldsymbol{\Lambda}_{L+R}(\mathbf{u})=\underset{j=1}{\times} \underset{1 \leq i \leq d}{\times}\left[\mathrm{u}_{j}^{(i)}-L-R, \mathrm{u}_{j}^{(i)}+L+R\right],
$$

cf. (1.5), (1.6). For $\boldsymbol{\Lambda}^{\prime}=\boldsymbol{\Lambda}_{L^{\prime}}\left(\mathbf{u}^{\prime}\right)$ the box $\widehat{\boldsymbol{\Lambda}}^{\prime}$ is constructed in the similar fashion. Here $R$ is the constant from Condition (C3) and vector $\underline{1}=(1, \ldots, 1)$. The projections $\Pi_{j} \widehat{\boldsymbol{\Lambda}}$ and $\Pi_{j} \widehat{\boldsymbol{\Lambda}}^{\prime}, j=1, \ldots, N$, are defined in the same way as for $\boldsymbol{\Lambda}$ and $\boldsymbol{\Lambda}^{\prime}$, and we set

$$
\Pi \widehat{\boldsymbol{\Lambda}}_{L}(\mathbf{u})=\bigcup_{j=1}^{N} \Pi_{j} \widehat{\boldsymbol{\Lambda}}, \quad \Pi \widehat{\boldsymbol{\Lambda}}^{\prime}(\mathbf{u})=\bigcup_{j=1}^{N} \Pi_{j} \widehat{\mathbf{\Lambda}}^{\prime}
$$

Since $R$ is a constant, the ratio of volumes $|\boldsymbol{\Lambda}|$ and $|\widehat{\Lambda}|$ is uniformly bounded from above and below when $L \geq 1$; the same is true for $\left|\boldsymbol{\Lambda}^{\prime}\right|$ and $\left|\widehat{\boldsymbol{\Lambda}}^{\prime}\right|$ when $L^{\prime} \geq 1$.

Definition 2 Fix a non-negative number $R$. We say that boxes $\boldsymbol{\Lambda}$ and $\boldsymbol{\Lambda}^{\prime}$ are separated (or $R$-separated) if, for some non-empty set $\mathcal{J} \subseteq\{1, \ldots, N\}$, either

$$
\operatorname{dist}\left[\left(\bigcup_{j \in \mathcal{J}} \Pi_{j} \widehat{\boldsymbol{\Lambda}}^{\prime}\right),\left(\bigcup_{l \notin \mathcal{J}} \Pi_{l} \widehat{\boldsymbol{\Lambda}}^{\prime} \cup \Pi \widehat{\boldsymbol{\Lambda}}\right)\right]>2 R
$$

or

$$
\operatorname{dist}\left[\left(\bigcup_{j \in \mathcal{J}} \Pi_{j} \widehat{\boldsymbol{\Lambda}}\right),\left(\bigcup_{l \notin \mathcal{J}} \Pi_{l} \widehat{\boldsymbol{\Lambda}} \cup \Pi \widehat{\boldsymbol{\Lambda}^{\prime}}\right)\right]>2 R .
$$

Example Let $d=1$ and $N=2$ (two one-dimensional particles), and $R=1$. Set

$$
\boldsymbol{\Lambda}=[2,3] \times[0,1], \quad \boldsymbol{\Lambda}^{\prime}=[6,7] \times[0,1] .
$$


We have:

$$
\Pi_{1} \boldsymbol{\Lambda}=[2,3], \quad \Pi_{2} \boldsymbol{\Lambda}=[0,1] ; \quad \Pi \mathbf{\Lambda}=[0,1] \cup[2,3] .
$$

Correspondingly,

$$
\Pi_{1} \mathbf{\Lambda}^{\prime}=[6,7], \quad \Pi_{2} \mathbf{\Lambda}^{\prime}=[0,1] ; \quad \Pi \mathbf{\Lambda}^{\prime}=[0,1] \cup[6,7] .
$$

One can see that squares $\boldsymbol{\Lambda}, \boldsymbol{\Lambda}^{\prime}$ are separated (namely, $R$-separated with $R=1$ ). Indeed, set $\mathcal{J}=\{1\}$, then we have

$$
\bigcup_{j \in \mathcal{J}} \Pi_{j}{\widehat{\boldsymbol{\Lambda}^{\prime}}}^{\prime}=\Pi_{1} \boldsymbol{\Lambda}^{\prime}=[6,7]
$$

while

$$
\bigcup_{l \notin \mathcal{J}} \Pi_{l} \widehat{\boldsymbol{\Lambda}}^{\prime} \cup \Pi \widehat{\boldsymbol{\Lambda}}=\Pi_{2} \boldsymbol{\Lambda}^{\prime} \cup \Pi \widehat{\boldsymbol{\Lambda}}=[0,1] \cup[2,3] \subset[0,3],
$$

so that, in this example,

$$
\operatorname{dist}\left[\left(\bigcup_{j \in \mathcal{J}} \Pi_{j} \widehat{\boldsymbol{\Lambda}}^{\prime}\right),\left(\bigcup_{l \notin \mathcal{J}} \Pi_{l} \widehat{\boldsymbol{\Lambda}}^{\prime} \cup \Pi \widehat{\boldsymbol{\Lambda}}\right)\right] \geq \operatorname{dist}[[0,3],[6,7]]>2=2 R .
$$

Physically speaking, the potential of the two-particle system corresponding to $\boldsymbol{\Lambda}$ is not affected by the values of the external random potential $V(x)$ taken at $x \in[6,7]$, while the potential energy of the system corresponding to $\boldsymbol{\Lambda}^{\prime}$ has a contribution of the form $V\left(x_{2}\right)$, with $x_{2} \in[6,7]$. Fixing the values of the random potential $V(x)$ for $x \in[0,1] \cup[2,3]$, we fix completely the potential energy of the system in $\boldsymbol{\Lambda}$, while the energy in $\boldsymbol{\Lambda}^{\prime}$ can still be varied by varying values $V(x)$ with $x \in[6,7]$. This "freedom", although more limited than in the conventional, one-particle localisation theory, turns out to be sufficient for the purposes of eigenvalue concentration bounds addressed in this paper.

Lemma 2 Let $R>0$ be the range of alloy-type potential $V(x, \omega)$ (cf. Condition (C3), (2.4)). Consider $2 R$-separated boxes $\boldsymbol{\Lambda}$ and $\boldsymbol{\Lambda}^{\prime}$ and let $\mathcal{J}$ be a non-empty subset in $\{1, \ldots, N\}$ figuring in Definition 2. Assume for definiteness that (4.2) holds true. Then

(A)

$$
\begin{aligned}
& \text { the family of random variables }\left(\mathrm{V}_{s}, s \in \bigcup_{j \in \mathcal{J}} \Pi_{j} \mathbf{\Lambda}^{\prime}\right) \text { is } \\
& \text { disjoint from the family }\left(\mathrm{V}_{t}, t \in \bigcup_{l \notin \mathcal{J}} \Pi_{l} \mathbf{\Lambda}^{\prime} \cup \Pi \mathbf{\Lambda}\right)
\end{aligned}
$$

(B) Moreover, the sigma-algebras generated by random variables of the form

$$
\left(\sum_{j \in \mathcal{J}} V\left(x_{j}, \omega\right), \mathbf{x} \in \mathbf{\Lambda}\right)
$$

and

$$
\left(\sum_{j \notin \mathcal{J}} V\left(x_{j}, \omega\right), \mathbf{x} \in \mathbf{\Lambda} ; \sum_{j=1}^{N} V\left(y_{j}, \omega\right), \mathbf{y} \in \mathbf{\Lambda}^{\prime}\right)
$$

are independent. 
Proof (A) By virtue of inequality (4.2), the $R$-neighbourhoods of the sets $\left(\bigcup_{j \in \mathcal{J}} \Pi_{j} \mathbf{\Lambda}^{\prime}\right)$ and $\left(\bigcup_{l \notin \mathcal{J}} \Pi_{l} \boldsymbol{\Lambda}^{\prime} \cup \Pi \mathbf{\Lambda}\right)$ are disjoint, and so are the two sets of indices $s$ figuring in (4.4). Therefore, the two families of random variables in question, labelled by two disjoint sets of indices, are also disjoint.

(B) Since the bump functions $\varphi$ have the support of radius $\leq R$, the potential $V(x, \omega)$ can be represented as follows:

$$
V(x, \omega)=\sum_{s \in \mathbb{Z}^{d}} \mathrm{~V}_{s}(\omega) \varphi\left(x_{j}-s\right)=\sum_{s \in \mathbb{Z}^{d}:\left\|s-x_{j}\right\| \leq R} \mathrm{~V}_{s}(\omega) \varphi\left(x_{j}-s\right),
$$

so that no random variable $\mathrm{V}_{\mathrm{s}}$ can simultaneously affect both a sum of the form (4.5) and (4.6). This implies assertion (B).

Theorem 2 Assume that Conditions (C0)-(C4) (see (1.2), (2.2)-(2.5)) are satisfied. Then, for every finite interval $I \subset \mathbb{R}$, there exists a constant $C_{2}=C_{2}(I)>0$ such that for every pair $\boldsymbol{\Lambda}$ and $\boldsymbol{\Lambda}^{\prime}$ of separated boxes and every $\epsilon \in(0,1)$ we have that

$$
\begin{aligned}
& \mathbb{P}\left(\operatorname{dist}\left[\Sigma\left(H_{\boldsymbol{\Lambda}}\right) \cap I, \Sigma\left(H_{\mathbf{\Lambda}^{\prime}}\right) \cap I\right] \leq \epsilon\right) \\
& \quad \leq C_{2}\left|\boldsymbol{\Lambda} \| \mathbf{\Lambda}^{\prime}\right| \max _{\mathcal{J}: \emptyset \neq \mathcal{J} \subseteq\{1, \ldots, N\}} \max \left[\left|\bigcup_{j \in \mathcal{J}} \Pi_{j} \boldsymbol{\Lambda}\right|,\left|\bigcup_{j \in \mathcal{J}} \Pi_{j} \boldsymbol{\Lambda}^{\prime}\right|\right] v(2 \epsilon) .
\end{aligned}
$$

Remarks (1) The RHS of (4.7) can be simplified when $\boldsymbol{\Lambda}$ and $\boldsymbol{\Lambda}^{\prime}$ are boxes of identical sidelength $2 L$ : it then can be taken to be $C_{2} N L^{d(4 N+1)} \nu(2 \epsilon)$. On the other hand, (4.7) remains valid in the more general case where cubes $\boldsymbol{\Lambda}$ and $\boldsymbol{\Lambda}^{\prime}$ are replaced by parallelepipeds with different sidelengths in various directions.

(2) The separation condition (see (4.2), (4.3)) seems somewhat restrictive: it is tempting to replace it by the requirement that boxes $\widehat{\boldsymbol{\Lambda}}$ and $\widehat{\boldsymbol{\Lambda}}^{\prime}$ (or, effectively, original boxes $\boldsymbol{\Lambda}$ and $\boldsymbol{\Lambda}^{\prime}$ ) are sufficiently distant apart. In fact, this can be done when $N=2$ (a two-particle system); see [8]. For $N \geq 3$, the separation condition in general is not implied when $\widehat{\boldsymbol{\Lambda}}$ and $\widehat{\boldsymbol{\Lambda}}^{\prime}$ are moved away from each other. However, it holds for 'almost all' positions of boxes $\boldsymbol{\Lambda}$ and $\boldsymbol{\Lambda}^{\prime}$ when they are sufficiently far away from each other. For details, see [10], Lemma 1.1. As was mentioned above, this suffices for applicability of the MSA for proving multi-particle Anderson localisation (which is our goal, as far as the Hamiltonian (1.1) is concerned, with the external field as in (1.3), (2.1)).

(3) Conditions (4.2), (4.3) can be relaxed. We use these conditions in the form given above for the reason that it leads to a straightforward proof of Lemma 2.

Proof of Theorem 2 As before, let $\mathcal{J}$ be a set figuring in Definition 2, for $\boldsymbol{\Lambda}$ and $\boldsymbol{\Lambda}^{\prime}$. Now, assume that (4.3) holds and set $J=\left(\bigcup_{j \in \mathcal{J}} \Pi_{j} \widehat{\Lambda}\right) \cap \mathbb{Z}^{d}$. We estimate the probability in question by conditioning on $\mathfrak{B}_{\mathbb{Z}^{d} \backslash J}$ :

$$
\begin{aligned}
& \mathbb{P}\left(\operatorname{dist}\left[\Sigma\left(H_{\boldsymbol{\Lambda}}\right) \cap I, \Sigma\left(H_{\Lambda^{\prime}}\right) \cap I\right] \leq \epsilon\right) \\
& \quad=\mathbb{E}\left[\mathbb{P}\left(\operatorname{dist}\left[\Sigma\left(H_{\boldsymbol{\Lambda}}\right) \cap I, \Sigma\left(H_{\boldsymbol{\Lambda}^{\prime}}\right) \cap I\right] \leq \epsilon \mid \mathfrak{B}_{\mathbb{Z}^{d} \backslash J}\right)\right] .
\end{aligned}
$$

First, we bound the inner probability in the RHS of (4.8): there are at most $c_{2}|\boldsymbol{\Lambda}|$ (respectively, $c_{2}\left|\boldsymbol{\Lambda}^{\prime}\right|$ ) eigenvalues of $H_{\boldsymbol{\Lambda}}$ (respectively, $H_{\boldsymbol{\Lambda}^{\prime}}$ ) in the interval $I$, where $c_{2}=$ $c_{2}(R, I)>0$ is a constant coming from the Weyl's law; cf. the proof of Theorem 1. Namely, $c_{2}(R, I) \leq \widetilde{C}_{1}(R)(1+E \vee 0)^{\frac{d}{2}}$. Moreover, by Lemma 2, operator $H_{\Lambda^{\prime}}$ and consequently its 
eigenvalues are determined by the family $\mathbf{V}_{J}=\left(\mathrm{V}_{s}, s \in J\right)$. Let us label these eigenvalues $E_{k^{\prime}}^{\left(\boldsymbol{\Lambda}^{\prime}\right)}\left(\mathbf{V}_{\mathbb{Z}^{d} \backslash J}\right)$, with $k^{\prime}=0, \ldots, K^{\prime}$, where $k^{\prime} \leq c_{2}\left|\mathbf{\Lambda}^{\prime}\right|$. This yields:

$$
\begin{aligned}
& \mathbb{P}\left(\operatorname{dist}\left[\Sigma\left(H_{\boldsymbol{\Lambda}}\right) \cap I, \Sigma\left(H_{\mathbf{\Lambda}^{\prime}}\right) \cap I\right] \leq \epsilon \mid \mathfrak{B}_{\mathbb{Z}^{d} \backslash J}\right) \\
& \quad \leq \sum_{k}^{K} \sum_{k^{\prime}}^{K^{\prime}} \mathbb{P}\left(\left|E_{k}^{(\boldsymbol{\Lambda})}\left(\mathbf{V}_{J}\right)-E_{k^{\prime}}^{\left(\boldsymbol{\Lambda}^{\prime}\right)}\left(\mathbf{V}_{\mathbb{Z}^{d} \backslash J}\right)\right| \leq \epsilon \mid \mathfrak{B}_{\mathbb{Z}^{d} \backslash J}\right) \\
& \quad \leq \sum_{k}^{K} c_{2}\left|\mathbf{\Lambda}^{\prime}\right| \sup _{E \in I} \mathbb{P}\left(\left|E_{k}^{(\boldsymbol{\Lambda})}\left(\mathbf{V}_{J}\right)-E\right| \leq \epsilon \mid \mathfrak{B}_{\mathbb{Z}^{d} \backslash J}\right) \\
& \quad=\sum_{k}^{K} c_{2}\left|\mathbf{\Lambda}^{\prime}\right| \sup _{E \in I} \mathbb{P}\left(E_{k}^{(\boldsymbol{\Lambda})}\left(\mathbf{V}_{J}\right) \in[E-\epsilon, E+\epsilon] \mid \mathfrak{B}_{\mathbb{Z}^{d} \backslash J}\right) .
\end{aligned}
$$

As in the proof of Theorem 1, the probability

$$
\mathbb{P}\left(E_{k}^{(\boldsymbol{\Lambda})}(\omega) \in[E-\epsilon, E+\epsilon] \mid \mathfrak{B}_{\mathbb{Z}^{d} \backslash J}\right) \leq c_{2}|\boldsymbol{\Lambda}|(\# J) \nu(\epsilon)
$$

which leads to the desired estimate (4.7).

Acknowledgements The authors thank The Isaac Newton Institute, University of Cambridge, for hospitality during the programme "Mathematics and Physics of the Anderson Localisation: 50 years after" (JulyDecember, 2008). The authors thank the referees for careful reading of the earlier version of tis manuscript and numerous remarks. P.S. thanks Université Paris VII and the DFG for supporting travel. P.S. also thanks H. Zenk for clarifying discussions on $[19,26]$. Y.S. thanks IHES, Bures-sur-Yvette, for hospitality during visits in 2008 .

\section{References}

1. Aizenman, M., Germinet, F., Klein, A., Warzel, S.: On Bernoulli decomposition for random variables, concentration bounds, and spectral localization. Probab. Theory Relat. Fields 143, 219-238 (2009)

2. Aizenman, M., Warzel, S.: On the joint distribution of energy levels of random Schrödinger operators. arXiv:0804.4231

3. Aizenman, M., Warzel, S.: Localization bounds for multiparticle systems. Commun. Math. Phys. 290, 903-934 (2009)

4. Boutet de Monvel, A., Chulaevsky, V., Stollmann, P., Suhov, Y.: Anderson localization in the continuous two-particle model with random potential. In preparation

5. Bourgain, J., Kenig, C.: On localization in the continuous Anderson-Bernoulli model in higher dimensions. Invent. Math. 161(2), 389-426 (2005)

6. Carmona, R., Lacroix, J.: Spectral Theory of Random Schrödinger Operators. Birkhäuser, Basel (1990)

7. Chulaevsky, V.: A Wegner-type bound for correlated potentials. Math. Phys. Anal. Geom. 11(2), 117$129(2008)$

8. Chulaevsky, V., Suhov, Y.: Wegner bounds for a two-particle tight binding model. Commun. Math. Phys. 283, 479-489 (2008)

9. Chulaevsky, V., Suhov, Y.: Eigenfunctions in a two-particle Anderson tight binding model. Commun. Math. Phys. 289, 701-723 (2009)

10. Chulaevsky, V., Suhov, Y.: Multi-particle Anderson localisation: induction in the number of particles. Math. Phys. Anal. Geom. 12(2), 117-139 (2009)

11. Combes, J.-M., Hislop, P.D., Klopp, F.: An optimal Wegner estimate and its application to the global continuity of the integrated density of states for random Schrödinger operators. Duke Math. J. 140(3), 469-498 (2007)

12. Durrett, R.: Probability: Theory and Examples. Duxbury, N. Scituate (1996)

13. Von Dreifus, E., Klein, A.: A new proof of localization in the Anderson tight binding model. Commun. Math. Phys. 124, 285-299 (1989) 
14. Edgar, G.A.: Disintegration of measures and the vector-valued Radon-Nikodỳm theorem. Duke Math. J. 42, 447-450 (1975)

15. Feller, W.: An Introduction to Probability Theory and Its Applications, vol. 2. Wiley, New York (1971)

16. Grimmett, G.R., Stirzaker, D.R.: Probability and Random Processes. Oxford University Press, London (2001)

17. Kirsch, W.: A Wegner estimate for multi-particle random Hamiltonians. J. Math. Phys. Anal. Geom. 4, 121-127 (2008)

18. Kirsch, W., Metzger, B.: The integrated density of states for random Schrödinger operators. In: Gesztesy, F. (Managing ed.), Deift, P., Galvez, C., Perry, P., Schlag, W. (eds.) Spectral Theory and Mathematical Physics: A Festschrift in Honor of Barry Simon's 60th Birthday. Ergodic Schrödinger Operators, Singular Spectrum, Orthogonal Polynomials, and Inverse Spectral Theory. Proc. Symp. Pure Math., vol. 76, Part 2, pp. 649-696. American Mathematical Society, Providence (2007)

19. Klopp, F., Zenk, H.: The integrated density of states for an interacting multielectron homogeneous model and applications to the Anderson model. Adv. Math. Phys. 2009, Article ID 6798727, 15 pages (2009). doi: $10.1155 / 2009 / 679827$

20. Pastur, L., Figotin, A.: Spectra of Random and Almost-periodic Operators. Springer, Berlin (1992)

21. Reed, M., Simon, B.: Methods of Modern Mathematical Physics, vol. 4. Academic Press, New York (1978)

22. Stollmann, P.: Wegner estimates and localization for continuous Anderson models with some singular distributions. Arch. Math. 75, 307-311 (2000)

23. Stollmann, P.: Caught by Disorder: Bound States in Random Media. Progress in Math. Phys., vol. 20. Birkhäuser, Basel (2001)

24. Veselić, I.: Existence and Regularity Properties of the Integrated Density of States of Random Schrödinger Operators. Lect. Notes Math., vol. 1917. Springer, Berlin (2008)

25. Wegner, F.: Bounds on the density of states in disordered systems. Z. Phys. B 44, 9-15 (1981)

26. Zenk, H.: An interacting multielectron Anderson model. Preprint ma.utexas.edu/mp_arc/c/03/03-410 